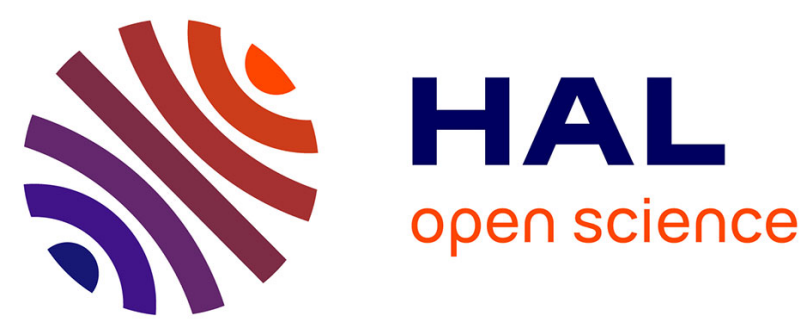

\title{
The Cooper problem in nanoscale: enhancement of the coupling due to confinement
}

\author{
Mihail D. Croitoru, A. Vagov, A. A. Shanenko, V. M. Axt
}

\section{To cite this version:}

Mihail D. Croitoru, A. Vagov, A. A. Shanenko, V. M. Axt. The Cooper problem in nanoscale: enhancement of the coupling due to confinement. Superconductor Science and Technology, 2012, 25 (12), pp.124001. 10.1088/0953-2048/25/12/124001 . hal-00772403

\section{HAL Id: hal-00772403 \\ https://hal.science/hal-00772403}

Submitted on 28 Aug 2018

HAL is a multi-disciplinary open access archive for the deposit and dissemination of scientific research documents, whether they are published or not. The documents may come from teaching and research institutions in France or abroad, or from public or private research centers.
L'archive ouverte pluridisciplinaire HAL, est destinée au dépôt et à la diffusion de documents scientifiques de niveau recherche, publiés ou non, émanant des établissements d'enseignement et de recherche français ou étrangers, des laboratoires publics ou privés.

\section{(1)(1) $\$(0)$}

Distributed under a Creative Commons Attribution - NonCommercial - ShareAlikel 4.0 


\title{
The Cooper problem in nanoscale: enhancement of the coupling due to confinement
}

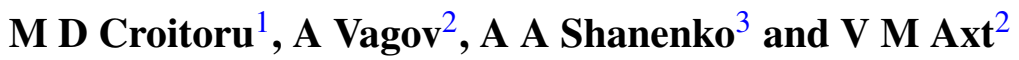 \\ ${ }^{1}$ Université Bordeaux I, LOMA, UMR 5798, F-33400 Talence, France \\ ${ }^{2}$ Institut für Theoretische Physik III, Universität Bayreuth, D-95440 Bayreuth, Germany \\ ${ }^{3}$ Departement Fysica, Universiteit Antwerpen, Groenenborgerlaan 171, B-2020 Antwerpen, Belgium \\ E-mail: mihail.croitoru@u-bordeaux1.fr
}

\begin{abstract}
In 1956 Cooper demonstrated (1956 Phys. Rev. 104 1189) that, no matter how weak the attraction is, two electrons in three-dimensional (3D) space just above the Fermi sea could be bound. In this work we investigate the influence of confinement on the binding energy of a Cooper pair. We show that confinement-induced modification of the Fermi sea results in a significant increase of the binding energy, when the bottom of an energy subband is very close to the Fermi surface.
\end{abstract}

(Some figures may appear in colour only in the online journal)

As was indicated by Cooper the ground state of the 3D electron gas, i.e. the filled Fermi sphere, is unstable if there is even an infinitesimally small electron-electron attraction near the Fermi surface [1]. Instead, a qualitatively different ground state appears in this system. By considering two electrons above the Fermi sea he found that these electrons are bound, and that the largest binding energy is

$$
E_{0}^{3 \mathrm{D}}=-2 \hbar \omega_{\mathrm{D}} \exp \left(-\frac{2}{|g| \nu_{\mathrm{F}}}\right)
$$

for two electrons having opposite momenta $(\mathbf{k},-\mathbf{k})$ and anti-parallel spins $(\uparrow, \downarrow)$. Here $\omega_{\mathrm{D}}$ is the Debye frequency, $g$ is a negative constant characterizing the attraction, and $\nu_{\mathrm{F}}$ is the electron density of states per spin at the chemical potential $\mu$. If electrons having nearly opposite momenta $(\mathbf{k},-\mathbf{k}+\mathbf{q})$ are paired ( $\mathbf{q}$ is the total momentum of a Cooper pair), the binding energy is less than $E_{0}$.

The degeneracy of the electron gas (Pauli exclusion principle) is the essential property of the system. Together with the attractive interaction between the particles it is the origin of the Fermi sea instability. It is well known that in the bulk (3D) case a weak attractive potential alone cannot create a bound state [2]. To form a bound state the attraction should be larger than a certain threshold. In contrast, in one- and two-dimensional cases a bound state can be created by an arbitrarily weak attractive potential. It is the filled Fermi sphere at low temperatures that transforms the 3D system to an effectively $2 \mathrm{D}$ one. Indeed, the Pauli exclusion principle prohibits the electrons from being scattered inside the Fermi sphere. Besides, the energy transfer between electrons due to the phonon exchange is of the order of $\hbar \omega_{\mathrm{D}}$, which is much smaller than the Fermi energy, $\mu$. Therefore electrons are effectively confined in a thin shell around the Fermi surface, if $\hbar \omega_{\mathrm{D}} \ll \mu$ [3]. This result of Cooper made it possible to conclude that the instability of the normal phase, driven by the Pauli exclusion principle and the effective electron-electron correlations is responsible for the occurrence of the superconducting phase $[4,5]$.

In recent years, due to remarkable experimental progress [6-18], the study of the superconducting properties in nanoscale has attracted a lot of interest [19-33]. A characteristic feature of such a system is quantum confinement of charge carriers. It results in important qualitative changes in the characteristics of superconductors with nanoscale dimensions, namely, any superconducting quantity (the superconducting order parameter, the energy gap, the critical temperature [23, 24], critical magnetic field [25], critical current [26], coherence length [27]) 
exhibits quantum-size oscillations with enhancement [9] and reduction [18] trends. In quasi-low-dimensional structures, the spatial distribution of the pair condensate exhibits distinct nonuniform patterns leading to the formation of the Andreev-type states. This significantly modifies the ratio of the energy gap to the critical temperature [32]. The superconducting-to-normal transition driven by a magnetic field becomes a cascade of first-order transitions contrary to the second-order transition found in the mesoscopic regime [25].

The goal of the present paper is to investigate the influence of quantum confinement on the binding energy in the single bound-pair model. The main feature of low-dimensional structures that makes them different from a bulk is the formation of a series of single-electron energy subbands (discrete electron levels in the case of quasi-0D). While the specimen thickness increases (decreases), these subbands move down (up) in energy.

Following Cooper, we consider two quasi-particles above the filled Fermi sphere. We assume translational invariance along one, e.g. the $z$-direction. We can write the two-particle wavefunction as

$$
\Psi\left(\mathbf{r}_{1}, \mathbf{r}_{2}\right)=\sum_{k} \sum_{i, j} \psi_{k i j} \mathrm{e}^{\mathrm{i} k\left(z_{1}-z_{2}\right)} \varphi_{i}\left(\rho_{1}\right) \varphi_{i}\left(\rho_{2}\right),
$$

with $i, j$ the quantum numbers associated with $\rho_{1}$ and $\rho_{2}$, respectively, and $k$ the wavevector of the quasi-free electron motion along the $z$-direction. Here we shall concentrate on the situation where the pair is at rest $(q=0)$ [3]. In this case the scattering phase volume is largest. The two-particle Schrödinger equation reads

$$
\left(E-\hat{H}_{0}\right) \Psi\left(\mathbf{r}_{1}, \mathbf{r}_{2}\right)=V\left(\mathbf{r}_{1}-\mathbf{r}_{2}\right) \Psi\left(\mathbf{r}_{1}, \mathbf{r}_{2}\right),
$$

where $\hat{H}_{0}=\frac{-\hbar^{2}}{2 m}\left(\nabla_{1}^{2}+\nabla_{2}^{2}\right)$. We make an approximation, that the potential $V\left(\mathbf{r}_{1}-\mathbf{r}_{2}\right)$ couples only electrons from time-reversed states. We can make this assumption because according to Anderson [34] the pairing of the time-reversed states is a good approximation in bulk in the absence of a magnetic field. For nanoscale systems, the Anderson's approximation is good enough provided that the pairing of two electrons from different single-electron subbands plays a minor role. As shown in [26] this is true when the subband-dependent pairing is much less than the subband energy spacing. We remark that this approximation becomes exact only in the situation of the spatially independent pairing. Our previous numerical investigations [26] have shown that the use of Anderson's recipe is well justified in nanoscale, i.e. corrections were found to be even less than a few per cent. Even for a broken time-reversal symmetry it was shown that Anderson's approximation still provides very good results for a cylindrical superconducting nanowire in a parallel magnetic field [25], because the single-electron wavefunctions remain unaltered in the linear order in the vector potential [25]. Within the Anderson approximation the form of the wavefunction, shown in equation (2) can be simplified, namely

$$
\Psi\left(\mathbf{r}_{1}, \mathbf{r}_{2}\right)=\sum_{k} \sum_{i} \psi_{k i} \mathrm{e}^{\mathrm{i} k\left(z_{1}-z_{2}\right)} \varphi_{i}\left(\rho_{1}\right) \varphi_{i}\left(\rho_{2}\right) .
$$

Substituting equation (4) into (3) and making projections on both sides of the equation, we obtain the following matrix equation

$$
\left(E-2 \varepsilon_{i}-\frac{\hbar^{2} k^{2}}{m}\right) \psi_{k i}=\sum_{q} \sum_{j} V_{k i, q j} \psi_{q j},
$$

where $V_{k i, q j}=\left\langle k, i ;-k, i\left|V\left(\mathbf{r}_{1}-\mathbf{r}_{2}\right)\right| q, j ;-q, j\right\rangle$. This equation is solved with $V\left(\mathbf{r}_{1}-\mathbf{r}_{2}\right)$ factorized as

$$
\left\langle k, i ;-k, i\left|V\left(\mathbf{r}_{1}-\mathbf{r}_{2}\right)\right| q, j ;-q, j\right\rangle=-\frac{g}{V} \omega_{k i}^{*} \omega_{q j},
$$

where the interaction is confined to the area very close to the Fermi surface

$$
\left|\omega_{k i}\right|^{2}=\Xi\left(\xi_{k, i}\right),
$$

with $\xi_{k, i} \equiv \varepsilon_{i}+\frac{\hbar^{2} k^{2}}{2 m}-\mu$, the energy spectrum of an electron in the Fermi sea, measured from the Fermi surface, $\mu, g$ is the coupling and $V$ is the volume of the system. Here $\Xi(\xi)=1$ if $\hbar \omega_{\mathrm{D}}>\xi>0$ and $\Xi(\xi)=0$ in other cases. Summing over the quantum numbers $k, j$, we find the relation

$$
\begin{aligned}
\sum_{k} \sum_{i} \omega_{k i} \psi_{k i}= & -\frac{g}{V} \sum_{k} \sum_{i} \frac{\omega_{k i}^{*} \omega_{k i}}{E-2 \varepsilon_{i}-\frac{\hbar^{2} k^{2}}{m}} \\
& \times \sum_{q} \sum_{j} \omega_{q j} \psi_{q j} .
\end{aligned}
$$

This is the eigenvalue equation, we have to solve. By measuring the energy of the pair relative to the chemical potential, $E=2 \mu+E_{0}$, we rewrite this equation as follows

$$
\frac{V}{g}=\sum_{k} \sum_{i} \frac{\omega_{k i}^{*} \omega_{k i}}{2 \varepsilon_{i}+\frac{\hbar^{2} k^{2}}{m}-2 \mu-E_{0}} .
$$

When the bottom of a parabolic subband passes through the Fermi surface, the density of states $\nu_{F}$ increases abruptly, since the density of states per unit length in 1D for one spin projection at the Fermi surface reads as

$$
\nu_{1 \mathrm{D}}(E) \mathrm{d} E=\frac{1}{\pi}\left(\frac{m}{2 \hbar^{2}}\right)^{1 / 2} \sum_{i} \frac{\theta\left(E-\varepsilon_{i}\right)}{\left(E-\varepsilon_{i}\right)^{1 / 2}} \mathrm{~d} E,
$$

where $\theta\left(E-\varepsilon_{i}\right)$ is the Heaviside function. Note that the position of the bottom of any subband scales as $1 / D^{2}$, with $D$ the specimen thickness. As an example, in figures 1(a) and (b), the single-electron subbands are schematically plotted versus the wavevector of the quasi-free electron motion along a nanowire. The narrow range of energies for electrons involved in the interaction is highlighted with the patterned boxes. Throughout this paper the energy range $\left[\mu, \mu+\hbar \omega_{\mathrm{D}}\right]$ is referred to as the Debye window. In figure 1(a) an off-resonant situation is drawn, since the bottom edges of all subbands 1-3 are situated outside of the Debye window. When increasing the thickness of the sample, the subbands move down, and the edge of the third subband now is found in the vicinity of the Fermi level. The resonant regime develops, when a subband enters the Debye window and approaches the chemical potential $\mu$ (as shown in figure 1(b)). Size evolution of the system leads to a sequence of peaks in the average density of 


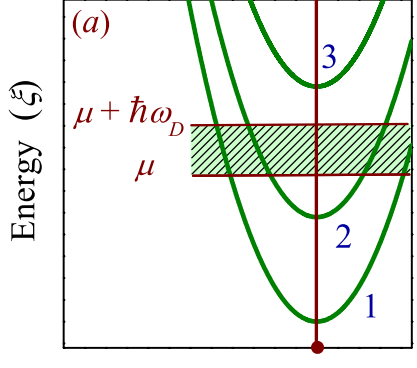

Wave vector $(k)$

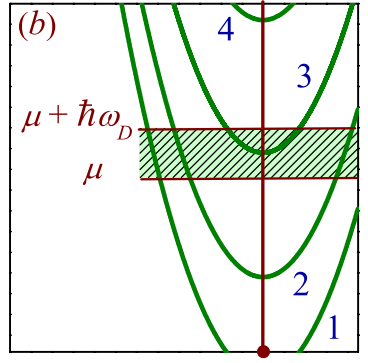

Wave vector $(k)$

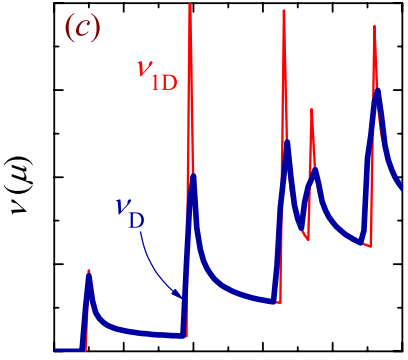

Thickness $(D)$

Figure 1. (a) Scheme of the single-electron subbands versus the wavevector of the motion parallel to the specimen for the off-resonant case. (b) The same as in panel (a) but for the resonant thickness. (c) The 1D density of states at the Fermi level versus the wire thickness $D$.

states $\nu_{\mathrm{D}}=\left(\hbar \omega_{\mathrm{D}}\right)^{-1} \int_{\mu}^{\mu+\hbar \omega_{\mathrm{D}}} \nu_{1 \mathrm{D}}(E) \mathrm{d} E$ as a function of the sample thickness $D$, as shown in figure 1(c). Here red thin and blue thick curves correspond to $v_{1 \mathrm{D}}$ and $\nu_{\mathrm{D}}$, respectively.

We can split the sum in equation (9) into two terms, namely

$$
\frac{V}{g}=\sum_{k} \sum_{i}^{\prime} \frac{\Xi\left(\xi_{k, i}\right)}{2 \xi_{k, i}-E_{0}}+\sum_{k} \frac{\Xi\left(\xi_{k, \mathrm{R}}\right)}{2 \xi_{k, \mathrm{R}}-E_{0}},
$$

where the prime means the absence of resonant subbands in the summation. Here it is supposed for simplicity that only one resonant subband is present in the spectrum that is labeled by $i=\mathrm{R}$. Considering $i$ as a continuous variable and making an approximation that the contribution from the non-resonant subbands to the density of states weakly depends on the energy in the Debye window, i.e. $v(E)=v(\mu)$, since for the non-resonant subbands $\hbar \omega_{\mathrm{D}} \ll \mu_{i} \equiv \mu-\varepsilon_{i}$, we can estimate the first term in equation (11) as

$$
F_{\mathrm{b}}=g \int_{0}^{\hbar \omega_{\mathrm{D}}} \frac{v(E) \mathrm{d} \xi}{2 \xi-E_{0}}=g \frac{\nu_{\mathrm{F}}}{2} \ln \left(\frac{E_{0}-2 \hbar \omega_{\mathrm{D}}}{E_{0}}\right),
$$

where $\nu_{\mathrm{F}}$ is the density of states per unit volume at energy $\mu$.

In the evaluation of the second term in equation (11), which we denote as $F_{\mathrm{c}}$, we make use of equation (10) for the density of states per unit length in $1 \mathrm{D}$. Then $F_{\mathrm{c}}$ acquires the following form

$$
F_{\mathrm{c}}=\frac{g}{\pi L^{2}}\left(\frac{m}{2 \hbar^{2}}\right)^{\frac{1}{2}} \int_{0}^{\hbar \omega_{\mathrm{D}}} \frac{\mathrm{d} \xi}{2 \xi-E_{0}} \frac{1}{\left(\xi-\xi_{\mathrm{R}}\right)^{1 / 2}},
$$

where $\xi_{R}=\varepsilon_{R}-\mu=-\mu_{R}$ is the energy of the bottom of the resonant subband with respect to the chemical potential and $L$ is the nanowire thickness. $\xi_{\mathrm{R}}$ is negative because we consider the regime where the bottom of the resonant subband is below the chemical potential. Performing the integration we get

$$
\begin{aligned}
F_{\mathrm{c}}= & \frac{g}{\pi L^{2}}\left(\frac{m}{\hbar^{2} \zeta}\right)^{\frac{1}{2}} \times\left[\operatorname{arctanh}\left(-\frac{2 \xi_{\mathrm{R}}}{\zeta}\right)^{\frac{1}{2}}\right. \\
& \left.-\operatorname{arctanh}\left(\frac{2 \hbar \omega_{\mathrm{D}}-2 \xi_{\mathrm{R}}}{\zeta}\right)^{\frac{1}{2}}\right]
\end{aligned}
$$

with $\zeta=E_{0}-2 \xi_{\mathrm{R}}=E_{0}+2 \mu_{\mathrm{R}}$. If the bottom edge of the subband $R$ is situated very close to the Fermi level, we can further approximate $\xi_{\mathrm{R}}=0$ and

$$
F_{\mathrm{c}}=\frac{g}{\pi L^{2}}\left(\frac{m}{\hbar^{2} E_{0}}\right)^{\frac{1}{2}} \operatorname{arctanh}\left(\frac{2 \hbar \omega_{\mathrm{D}}}{E_{0}}\right)^{\frac{1}{2}} .
$$

Since usually $\hbar \omega_{\mathrm{D}} \gg\left|E_{0}\right|$, and making use of

$$
\lim _{\hbar \omega_{\mathrm{D}} \gg\left|E_{0}\right|} \operatorname{arctanh}\left(-\frac{2 \hbar \omega_{\mathrm{D}}}{E_{0}}\right)^{\frac{1}{2}}=\frac{i \pi}{2},
$$

we get the total condition in the form

$$
\frac{1}{g}=\frac{\nu_{\mathrm{F}}}{2} \ln \left(\frac{E_{0}-2 \hbar \omega_{\mathrm{D}}}{E_{0}}\right)+\frac{1}{L^{2}}\left(-\frac{m}{4 \hbar^{2} E_{0}}\right)^{\frac{1}{2}} .
$$

Of course if there are $M$ resonance subbands, with bottom edges in the vicinity of the Fermi surface the second term should be multiplied by $M$.

If the system is in the bulk limit, $L \rightarrow \infty$, then the contribution of the second term is negligible and we obtain the Cooper expression for the binding energy (1). The reduction of dimensions of the system when approaching the nanoscale regime splits the conduction band into a multiple of subbands. Since confinement is weak, the quantization energy is small and the number of subbands with edges in the Debye window is large (see figure 2(c)). There is always a finite and large probability of finding the edge of a subband very close to the Fermi surface. This subband we call resonant. Since the number of subbands is large, the contribution of the resonant subband to the total density of states is small and can be considered as a perturbation. For the zero-order perturbation $F_{\mathrm{c}}=0$ and this leads to the bulk result of equation (1). For the first-order perturbation we obtain the Cooper result modified due to the influence of the resonant subband

$$
E_{0}^{1 \mathrm{D} \rightarrow 3 \mathrm{D}}=-2 \hbar \omega_{\mathrm{D}} \exp \left(-\frac{2}{|\widetilde{g}| \nu_{\mathrm{F}}}\right),
$$

where

$$
\widetilde{g}^{-1}=g^{-1}-\frac{1}{\hbar \nu_{\mathrm{F}} L^{2}}\left(\frac{m}{2 \hbar \omega_{\mathrm{D}}}\right)^{\frac{1}{2}} \exp \left(\frac{1}{|g| \nu_{\mathrm{F}}}\right) .
$$

Hence the binding energy of the pair increases exponentially as $L$ decreases. This is the origin of the sharp resonances.

In the case of strong confinement, the resonant subbands' contribution to the density of states is significant, even 

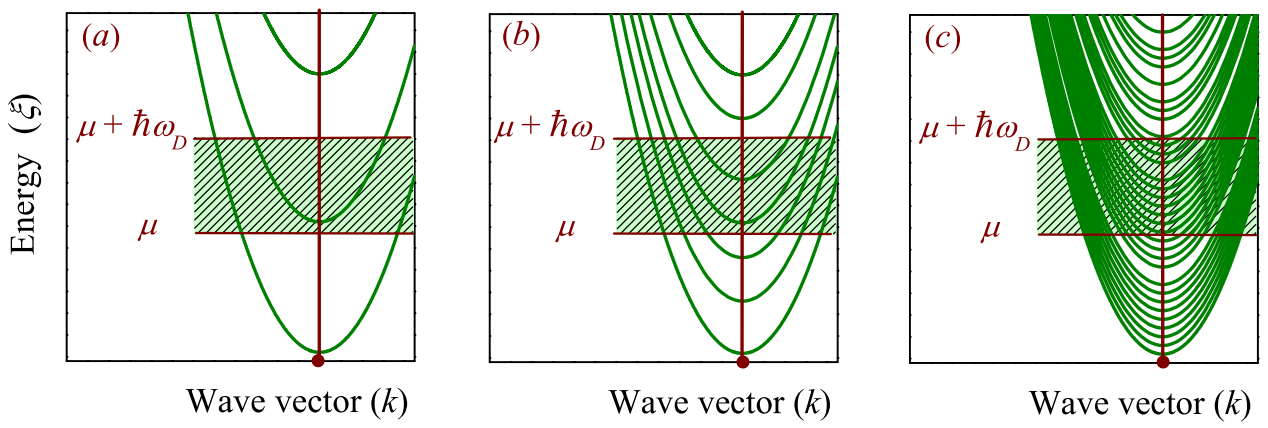

Figure 2. Scheme of the single-electron subbands versus the wavevector of the motion parallel to the specimen for (a) an ultra-small sample, (b) a sample with moderate confinement, (c) a weakly confined sample.

determinative (see figure 2(a)). When the edge of the resonant subband touches the Fermi surface the main contribution to the density of states comes from the second term in equation (17). The contribution from the other subbands with bottom edges below the Fermi surface can be viewed as a perturbation. In the zero-order approximation equation (17) reads as

$$
\frac{1}{g}=\frac{1}{L^{2}}\left(-\frac{m}{4 \hbar^{2} E_{0}}\right)^{\frac{1}{2}},
$$

which leads to the well-known $1 \mathrm{D}$ result for the energy of the bound state in the attractive $\delta$-function potential with the coupling constant $g / L^{2}$ (we have the extra factor $1 / 2$ due to the reduced mass $m / 2$ )

$$
E_{0}^{1 \mathrm{D}}=-\frac{m g^{2}}{4 \hbar^{2} L^{4}} .
$$

Making the same iterative procedure as above, we get the Cooper pair binding energy in the first-order perturbation

$$
E_{0}^{3 \mathrm{D} \rightarrow 1 \mathrm{D}}=-\frac{m g^{2}}{4 \hbar^{2} L^{4}\left[1-\frac{|g| \nu_{\mathrm{F}}}{2} \ln \left(\frac{8 \hbar^{3} \omega_{\mathrm{D}} L^{4}}{m g^{2}}\right)\right]^{2}} .
$$

The model presented here is oversimplified. For example it cannot describe a situation of moderate confinement, as presented in figure 2(b). Nevertheless it enables us to investigate the influence of the system's dimensionality on the binding energy of the Cooper pair in the presence of the Fermi sea. Notice that the binding energy obtained in the zero-order approximation, equation (21), is just the exact binding energy of two particles in a vacuum for the case of a 1D interaction model with $V(x)=-V_{0} \delta(x)$. Therefore, quantum confinement in the resonant-subband regime opens a channel for the real (molecule) binding. In other words, the presence of the resonant subband very near to the Fermi surface reconfigures the scattering phase space and forms an effective low-dimensional situation. The physics behind can be understood as follows. Two additional electrons can scatter via their interaction to all states of the same total momentum that are unoccupied by other electrons. The correlation between these electrons depends on the number of states in the Debye window above the Fermi sea available to form a pair [29]. When a subband approaches the Fermi level the number of states available for scattering strongly increases. This results in an increase of the binding energy. Since the Fermi sea in our model is frozen, i.e. there is no correlation between the electrons in the Fermi sea and two additional electrons, these two electrons can scatter only from the present state to all other empty states, but there is no in-scattering flow to these states. Besides, there is no possibility for these two electrons to scatter below the Fermi surface.

So far we have studied pairing in the situation of $\xi_{R}<0$. Let us consider another regime when the chemical potential is situated slightly below the bottom edge of the empty subband. We can also call this subband resonant and split the sum in equation (9), resulting in equation (11), because the contribution to the averaged density of states from this subband is larger than from the others. Hence, equation (11) reads as

$1=\frac{g}{2 \pi L^{2}}\left(\frac{2 m}{\hbar^{2}}\right)^{\frac{1}{2}} \int_{\xi_{\mathrm{R}}}^{\hbar \omega_{\mathrm{D}}} \frac{\mathrm{d} \xi}{2 \xi-E_{0}} \frac{1}{\left(\xi-\xi_{\mathrm{R}}\right)^{1 / 2}}+F_{\mathrm{b}}$.

In the zero-order perturbation, the integration of this equation gives

$$
1=\frac{g}{2 \pi L^{2}}\left(\frac{2 m}{\hbar^{2}}\right)^{\frac{1}{2}} \frac{\operatorname{arctanh}\left(\frac{\hbar \omega_{\mathrm{D}}-\xi_{\mathrm{R}}}{\left(\xi_{\mathrm{R}}-E_{0} / 2\right)}\right)^{\frac{1}{2}}}{\left(\xi_{\mathrm{R}}-E_{0} / 2\right)^{1 / 2}} .
$$

Assuming that $\hbar \omega_{\mathrm{D}} \gg|E|$ and $\hbar \omega_{\mathrm{D}} \gg \xi_{\mathrm{R}}$ we find that

$$
E_{0}=2 \xi_{\mathrm{R}}-\frac{m g^{2}}{4 \hbar^{2} L^{4}}
$$

The first term here is positive and the second is negative. While the bottom edge of the resonant subband moves up, the binding energy in this approximation decreases and finally it becomes zero, when $2 \xi_{\mathrm{R}}=\frac{m g^{2}}{4 \hbar^{2} L^{4}}$, meaning that the contribution of the resonant subband is no longer dominant and we have to take into account the contributions from the other subbands. In other words, we are back to the bulk solution for the Cooper pair problem: the resonance in the binding energy disappears. We remark that the same occurs when the bottom of the resonant subband goes far below the Fermi surface.

It is known that the spatial extent of the pair in the 3D Cooper problem is $\xi_{3 \mathrm{D}} \approx 1.15 \hbar v_{\mathrm{F}} / E_{0}$, which is $\sim 10^{4} \AA$. In 
the resonant regime, the pair binding is enhanced and it is expected that this enhancement should modify the size of the Cooper pair. Therefore, it is of interest to investigate the influence of quantum confinement on the size of a Cooper pair. Obviously, the size of the pair in the confined directions is determined by the size of the confinement. The size of the Cooper pair along the non-confined direction may be defined as

$$
\begin{aligned}
\xi_{\mathrm{C}}^{2} & =\frac{\int\left|\Psi\left(\rho_{1}, \rho_{2}, z\right)\right|^{2} z^{2} \mathrm{~d} z}{\int\left|\Psi\left(\rho_{1}, \rho_{2}, z\right)\right|^{2} \mathrm{~d} z} \\
& =\frac{\sum_{i} \varphi_{i}\left(\boldsymbol{\rho}_{\mathbf{1}}\right) \varphi_{i}\left(\boldsymbol{\rho}_{2}\right) \int\left|\frac{\partial}{\partial k} \psi_{k i}\right|^{2} \mathrm{~d} k}{\sum_{i} \varphi_{i}\left(\boldsymbol{\rho}_{\mathbf{1}}\right) \varphi_{i}\left(\boldsymbol{\rho}_{\mathbf{2}}\right) \int\left|\psi_{k i}\right|^{2} \mathrm{~d} k}
\end{aligned}
$$

If we neglect the contribution from the non-resonant subbands and consider in the summation only the resonant one, substituting equation (4) here we obtain

$$
\xi_{\mathrm{C}}^{2}=\frac{\int_{0}^{\hbar \omega_{\mathrm{D}}} \frac{\mathrm{d} \xi}{\left(2 \xi-E_{0}\right)^{4}}\left(\xi-\xi_{\mathrm{R}}\right)^{1 / 2}}{\int_{0}^{\hbar \omega_{\mathrm{D}}} \frac{\mathrm{d} \xi}{\left(2 \xi-E_{0}\right)^{2}} \frac{1}{\left(\xi-\xi_{\mathrm{R}}\right)^{1 / 2}}},
$$

where we have taken into account that

$$
\begin{aligned}
\left(E_{0}-2 \xi_{\mathrm{R}}\right) \psi_{k i} & =\sum_{q} \sum_{j} V_{k i, q j} \psi_{q j} \\
& =-\lambda \omega_{k, i}^{*} \sum_{q} \sum_{j} \omega_{q, j}=\mathrm{const},
\end{aligned}
$$

and performing the integration under the condition $\hbar \omega_{\mathrm{D}} \rightarrow \infty$ we get

$$
\xi_{\mathrm{C}}^{2}=\frac{\hbar^{2}}{8 m E_{0}} .
$$

Since in the resonance subband the binding energy $E_{0}$ is large, the size of the resonant Cooper pair becomes small. We can say that the Cooper pair becomes like a point boson dimer.

In summary, we solved the Cooper problem of two fermions attracting each other pairwise via a $3 \mathrm{D}$ potential in the presence of quantum confinement. We found that in the strong confinement regime the behavior of the binding energy is qualitatively different from that in the bulk. In the presence of quantum confinement the band of single-electron states in a clean nanosample is split up in a series of subbands. While the specimen thickness increases (decreases), these subbands move down (up) in energy. Each time when the bottom of a parabolic subband passes through the Fermi surface, the binding energy of a resonant Cooper pair strongly increases. The resonant Cooper pair becomes like a point dimer.

\section{Acknowledgment}

MDC acknowledges support by the European Community under the Marie Curie IEF Action (Grant Agreement No. PIEF-GA-2009-235486-ScQSR).

\section{References}

[1] Cooper L N 1956 Phys. Rev. 1041189

[2] Landau L D and Lifshitz L M 1977 Quantum Mechanics Non-Relativistic Theory 3rd edn, vol 3 (Oxford: Pergamon)

[3] Zagoskin A M 1998 Quantum Theory of Many-Body Systems: Techniques and Applications (New York: Springer)

[4] Bardeen J, Cooper L N and Schrieffer J R 1957 Phys. Rev. 108 1175-204

[5] Schrieffer J R 1999 Theory of Superconductivity (Massachusetts: Perseus)

[6] Özer M M, Thomson J R and Weitering H H 2006 Nature Phys. 2173

[7] Eom D, Qin S, Chou M-Y and Shih C K 2006 Phys. Rev. Lett. 96027005

[8] Savolainen M, Touboltsev V, Koppinen P, Riikonen K-P and Arutyunov K 2004 Appl. Phys. A 791769

[9] Zgirski M, Riikonen K-P, Touboltsev V and Arutyunov K 2005 Nano Lett. 51029

[10] Tian M, Wang J, Kurtz J S, Liu Y, Chan M H W, Mayer T S and Mallouk T E 2005 Phys. Rev. B 71104521

[11] Altomare F, Chang A M, Melloch M R, Hong Y and Tu C W 2006 Phys. Rev. Lett. 97017001

[12] Janković L et al 2006 Nano Lett. 61131

[13] Nishio T et al 2008 Phys. Rev. Lett. 101167001

[14] Wang K, Zhang X, Loy M M T, Chiang T-C and Xiao X 2009 Phys. Rev. Lett. 102076801

[15] Brun C, Po Hong I, Patthey F, Sklyadneva I Y, Heid R, Echenique P M, Bohnen K P, Chulkov E V and Schneider W D 2009 Phys. Rev. Lett. 102207002

[16] Qin S Y, Kim J, Niu Q and Shih C K 2009 Science 3241314

[17] Zhang T et al 2010 Nature Phys. 6104

[18] Bose S, García-García A M, Ugeda M M, Urbina J D, Michaelis C H, Brihuega I and Kern K 2010 Nature Mater. 9550

[19] Perali A, Bianconi A, Lanzara A and Saini N L 1996 Solid State Commun. 100181

[20] Gladilin V N, Fomin V M and Devreese J T 2002 Solid State Commun. 121519

[21] Gladilin V N, Fomin V M and Devreese J T 2004 Phys. Rev. B 70144506

[22] Ovchinnikov Y N and Kresin V Z 2005 Eur. Phys. J. B 455

Ovchinnikov Y N and Kresin V Z 2005 Eur. Phys. J. B 47333

[23] Shanenko A A and Croitoru M D 2006 Phys. Rev. B 73012510

[24] Croitoru M D, Shanenko A A and Peeters F M 2007 Phys. Rev. B 76024511

[25] Shanenko A A, Croitoru M D and Peeters F M 2008 Phys. Rev. B 78024505

[26] Croitoru M D, Shanenko A A, Kaun C C and Peeters F M 2009 Phys. Rev. B 80024513

[27] Shanenko A A, Croitoru M D, Vagov A and Peeters F M 2010 Phys. Rev. B 82104524

[28] Innocenti D, Poccia N, Ricci A, Valletta A, Caprara S, Perali A and Bianconi A 2010 Phys. Rev. B 82184528

[29] Pogosov W V, Combescot M and Crouzeix M 2010 Phys. Rev. B 81174514

[30] García-García A M, Urbina J D, Yuzbashyan E A, Richter K and Altshule B L 2011 Phys. Rev. B 83014510

[31] Croitoru M D, Shanenko A A, Kaun C C and Peeters F M 2011 Phys. Rev. B 83214509

[32] Croitoru M D, Shanenko A A, Peeters F M and Axt V M 2011 Phys. Rev. B 84214518

[33] Croitoru M D, Shanenko A A, Kaun C C and Peeters F M 2012 J. Phys.: Condens. Matter 24275701

[34] Anderson P W J 1959 J. Phys. Chem. Solids 1126 